\title{
Testing of a Microfluidic Sampling System for High Temperature Electrochemical MC\&A
}

Fuel Cycle Research \& Development

\author{
Prepared for \\ U.S. Department of Energy \\ MPACT Campaign \\ Candido Pereira \\ Kevin Nichols \\ Argonne National Laboratory \\ September 26, 2013
}




\section{DISCLAIMER}

This information was prepared as an account of work sponsored by an agency of the U.S. Government. Neither the U.S. Government nor any agency thereof, nor any of their employees, makes any warranty, expressed or implied, or assumes any legal liability or responsibility for the accuracy, completeness, or usefulness, of any information, apparatus, product, or process disclosed, or represents that its use would not infringe privately owned rights. References herein to any specific commercial product, process, or service by trade name, trade mark, manufacturer, or otherwise, does not necessarily constitute or imply its endorsement, recommendation, or favoring by the U.S. Government or any agency thereof. The views and opinions of authors expressed herein do not necessarily state or reflect those of the U.S. Government or any agency thereof. 


\section{Summary}

This report describes the preliminary validation of a high-temperature microfluidic chip system for sampling of electrochemical process salt. Electroanalytical and spectroscopic techniques are attractive candidates for improvement through high-throughput sample analysis via miniaturization. Further, microfluidic chip systems are amenable to microscale chemical processing such as rapid, automated sample purification to improve sensor performance. The microfluidic chip was tested to determine the feasibility of the system for high temperature applications and conditions under which microfluidic systems can be used to generate salt droplets at process temperature to support development of material balance and control systems in a used fuel treatment facility. In FY13, the project focused on testing a quartz microchip device with molten salts at near process temperatures. The equipment was installed in glove box and tested up to $400^{\circ} \mathrm{C}$ using commercial thermal transfer fluids as the carrier phase. Preliminary tests were carried out with a low-melting halide salt to initially characterize the properties of this novel liquid-liquid system and to investigate the operating regimes for inducing droplet flow within candidate carrier fluids. Initial results show that the concept is viable for high temperature sampling but further development is required to optimize the system to operate with process relevant molten salts. 
Testing of a Microfluidic Sampling System for High Temperature Electrochemical MC\&A September 26, 2013

\section{Contents}

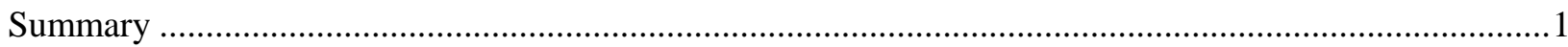

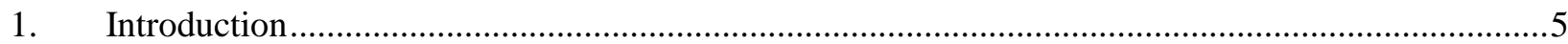

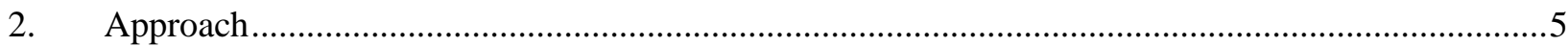

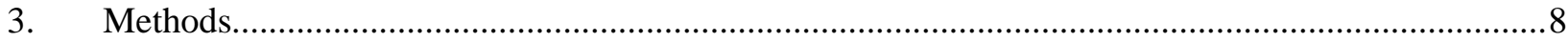

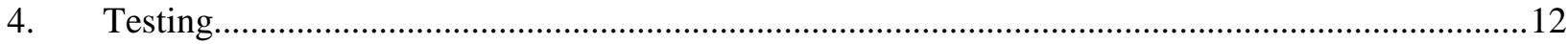

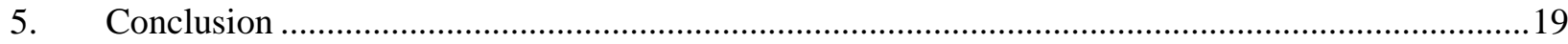

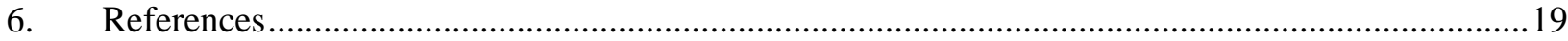




\section{Figures}

Figure 1: An example of a microfluidic chip developed at ANL for aqueous solvent extraction. 6

Figure 2: An aqueous/organic emulsion generated in an ANL microfluidic solvent extraction chip.

Figure 3: A schematic representation of the proposed microfluidic sampling system in relation to the electrorefiner and the cooled detector system.

Figure 4: A schematic of the electrochemical processing flow diagram with potential locations (stars) for salt sampling system to maintain a compositional mass balance............................

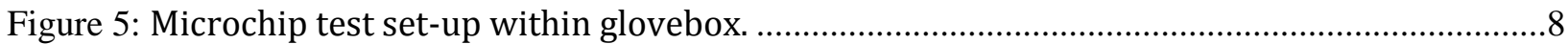

Figure 6: Experimental setup prior to insertion into the inert atmosphere glovebox. ........................9

Figure 7: Conceptual set-ups for droplet tests (a) static and (b) flowing..........................................11

Figure 8: Schematic of desired fluid flow within the microchip.

Figure 9: Dependency of Weber number as function of flow rate for different fluids at two linear velocities (a) low value and (b) higher value. The molten salts curves essentially overlap.

Figure 10: Photo of microchannel section showing the apparent two-phase fluid in flow

Figure 11: Photo of inlet cross-junction showing inflows of salt (top) and carrier fluid (sides) and resulting two-phase emulsion (bottom)

Figure 12: Set of photos showing different mixed flows of molten salt and heat transfer fluids within a microchannel (a) annular, (b) wavy parallel flow, (c) foamy solid salt (not flowing), and (d) bubbly flow....

Figure 13: Set of photos showing flow progression at inlet junction of salt (from top center) combining with heat transfer fluid (from top left and right) to form two phase wavy parallel flow (downward and bottom left).

Figure 14: Photo of frozen salt "droplet" within microchannel system

Figure 15: Photo showing two-phase slug flow in three microchannels. Consistent flow is evident in bottom two channels. Clear interfacial delineations suggest gas-liquid rather than liquid-liquid mixed flows 
Testing of a Microfluidic Sampling System for High Temperature Electrochemical MC\&A September 26, 2013

\section{Tables}



Table 2: Properties of Thermal Transfer Fluids .................................................................................10 


\section{Testing of a Microfluidic Sampling System for High Temperature Electrochemical MC\&A}

\section{Introduction}

Tracking the composition of salt as it moves through an electrochemical reprocessing facility is a difficult task because electrochemical material processing is done continuously at elevated temperatures while transfers involve discrete batches of used fuel or solids with adhered salts. Salt is recycled at several points within the process, making it difficult to relate changes in composition to specific processing operations. Particularly over long time steps, small differences or inhomogeneities may result in quantifiable changes in product output compositions. It is desirable to obtain an accurate and timely measure of the chemical and isotopic composition of the salt within the electrorefiner and through other operations in the facility to fully close the material mass balance.

The goal of this project is to develop a sampling system that will enable the accurate determination of the chemical and isotopic composition of the molten salt in an electrorefiner or adhering to cathode products or cladding wastes within a electrochemical facility. The system will ideally allow the combination of the best aspects of destructive analysis (high sensitivity, low measurement uncertainty, and high quantitative resolution) with the best aspect of non-destructive analysis (low sample loss, low processing impact, and rapid turnaround).

Practical safeguard difficulties associated with electrochemical processing include the absence of an input accountancy tank, high temperature processing, and batch-wise fuel addition and product harvesting coupled with synchronous dissolution and cathode deposition within the electrorefiner. In addition, salt is collected and recycled at several points within the cathode processing cycle, making it challenging to fully close the mass balance after the initial batch of fuel is dissolved. In addition, salt collected from processed cathodes may differ in composition from the balance of salt in the electrorefiner. Finally, minor inhomogeneity may exist in the salt or metal compositions in the electrorefiner.

\section{Approach}

Electroanalytical and spectroscopic techniques are attractive candidates for improvement through high-throughput sampling. Even the best analytical techniques can have issues with interfering signals, and variability arising from sample inhomogeneity must be addressed. Collection and measurement of a large number of samples, as is possible with microsamples, can reduce the statistical error associated with more traditional sample collection methods. Sample purification will also improve sensor performance, and microfluidic systems are very adaptable to common purification techniques (e.g. solvent extraction ${ }^{1}$ ), which can be automated at the micro-scale thus reducing the need for direct manipulation. 


\section{Testing of a Microfluidic Sampling System for High Temperature Electrochemical MC\&A \\ September 26, 2013 \\ For high temperature fluids, the reduced sample scale allows for contact with cooled detectors as droplets can be frozen in a carrier stream of a liquid that has a lower freezing point.}

To meet the project's goals, and overcome some of the identified difficulties associated with electrochemical safeguards, we are developing a microfluidic sampling system that can be coupled to traditional, room-temperature analytical equipment. It may also be feasible to deploy remote optical microanalytical systems that can function at a distance from the sample. Microfluidic systems are micromachined fluidic networks operating at low Reynolds and Weber numbers. Low Reynolds numbers allow highly repeatable, deterministic flow. Low Weber numbers allow the formation of multiphase mixtures in a fluidic regime that is resistant to clogging. ${ }^{1}$

A microfluidic network can provide a feasible means of obtaining near-simultaneous grab samples from multiple depths throughout the electrorefiner, and allows intimate contact with cooled detectors, while using a volume of material that is comparable with that lost using techniques typically considered non-destructive (such as Laser-induced Breakdown Spectroscopy - LIBS). A microchip system that uses a single-phase salt would lead to freezing and clogging within the microchannels before the detector is reached. A larger dimension multi-fluid system would lead to clogging due to the flow profiles present in higher Weber number systems, while a larger dimension salt collection system would require heated transfer lines and significantly larger amounts of salt to be transferred.

We have previously demonstrated microfluidic systems capable of performing solvent extraction unit operations on lanthanides and actinides. ${ }^{2}$ The sampling system proposed for electrochemical operations is an extension of this concept. Figures 1 and 2 show the microchip and droplet configurations for these aqueous microfluidic systems.

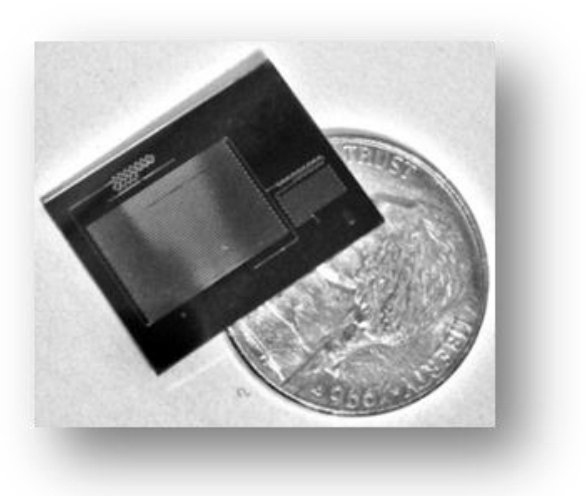

Figure 1 - An example of a microfluidic chip developed at ANL for aqueous solvent extraction.

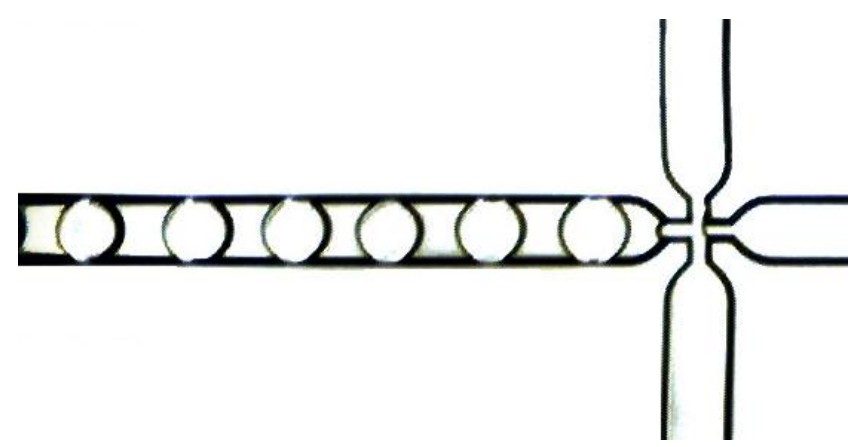

Figure 2 - An aqueous/organic emulsion generated in an ANL microfluidic solvent extraction chip.

The extension of ANL's droplet solvent extraction technique to molten salt systems is shown conceptually in Figure 3. In operation, a sample of in-process molten salt will be drawn via vacuum into a microfluidic channel network, superficially similar to that shown in Figure 2. With a properly designed channel, and operation at the correct Weber number ${ }^{3}$ the salt can be combined with an immiscible carrier phase in such a way that highly uniform droplets of salt are continuously formed in a flowing carrier phase. These droplets, and any suspended 


\section{Testing of a Microfluidic Sampling System for High Temperature Electrochemical MC\&A \\ September 26, 2013}

solids, are prevented from sticking and clogging via the fluid recirculation patterns associated with low Weber numbers. Additionally, as the droplets of molten salt cool, they will slightly contract, further facilitating transport.

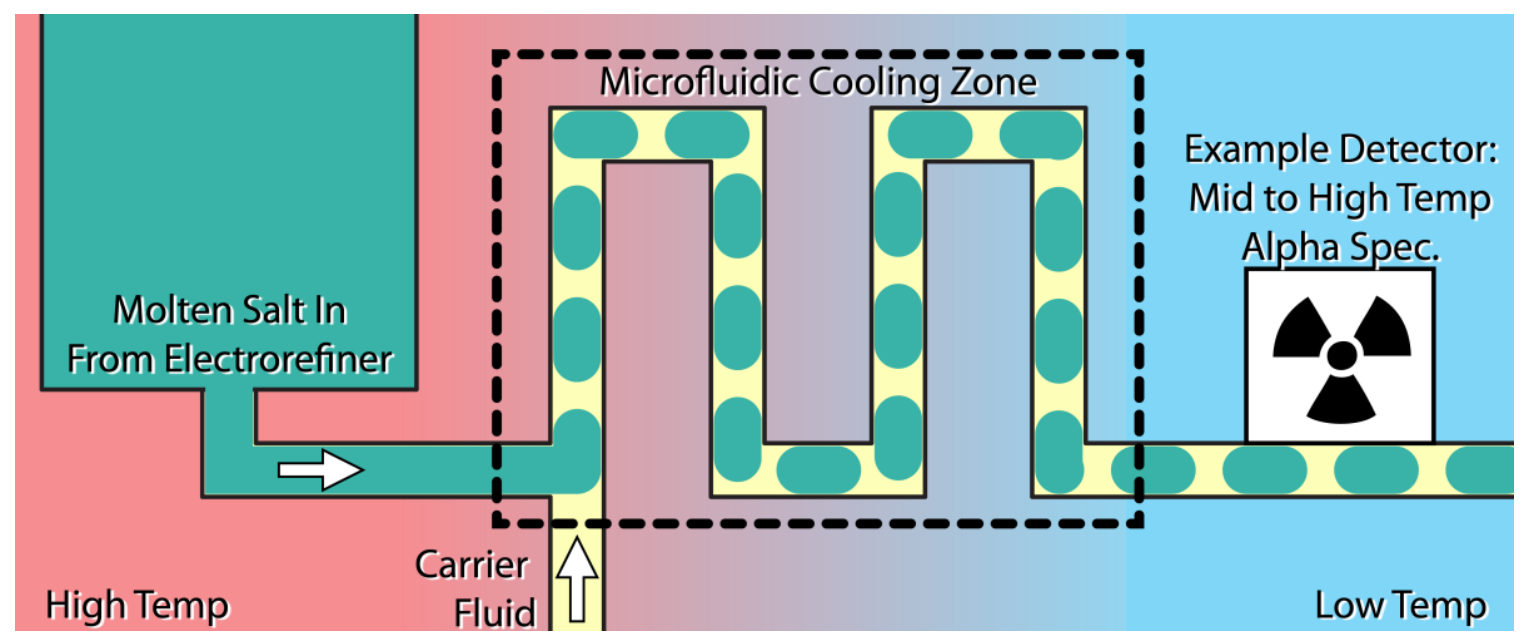

Figure 3 - A schematic representation of the proposed microfluidic sampling system in relation to the electrorefiner and the cooled detector system.

The microfluidic salt-sampling system may be deployed in a number of positions within the electrochemical reprocessing facility as shown in Figure 4. Whether samples are collected from all of these locations, or a subset, microfluidic-scale sampling enables interrogation of a large number of streams with relatively minimal impact on processing. One of the advantages of the droplet-based microfluidic approach is that the individual droplets are on the order of tens of nanoliters. Consequently, a large statistically significant number of discrete and consistent samples can be collected while the total quantity of material collected is on the order of milliliters.

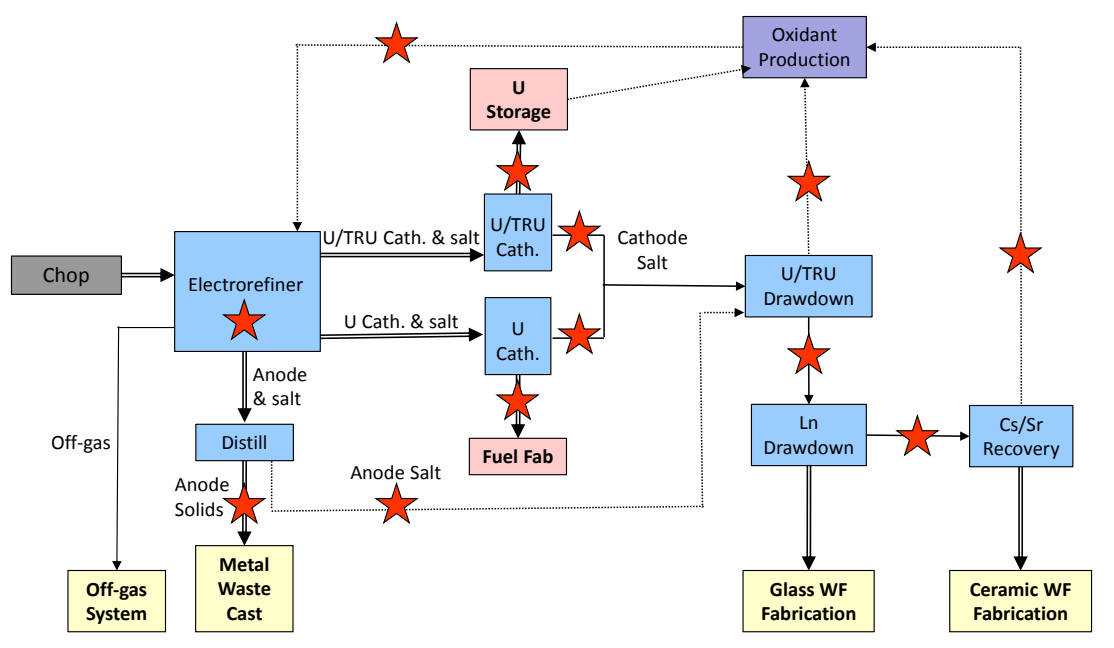

Figure 4 - A schematic of the electrochemical processing flow diagram with potential locations (stars) for salt sampling system to maintain a compositional mass balance. 
Testing of a Microfluidic Sampling System for High Temperature Electrochemical MC\&A

September 26, 2013

\section{Methods}

During FY13, the project was focused on testing the feasibility of producing two-phase flows consisting of uniform molten salt droplets in a carrier fluid confined within microchannels at elevated temperatures. Candidate heat transfer carrier fluids were identified and one was tested within a heated chip in order to characterize fluid flows and measure fluid properties. A long distance microscopy system was set-up for imaging the microflows in real time and at near-process temperature.

The high-temperature chip holder, gas flow, heater, camera and imaging systems procured in FY-12 were installed and assembled within an inert atmosphere glovebox. Figure 5 is a schematic of the set-up in the glovebox. Figure 6 shows the setup before it was placed in the glovebox. A nitrogen manifold was interfaced with a Mitos P-Pump, which regulated fluid flows within the chip channels in the glovebox. The data acquisition system was interfaced with a laptop computer within the glovebox; control software was installed for both the pump and the imaging systems. The inlet-gas flow control system and on-line software allow for rapid adjustment of liquid feed flow rates. A Nikon D7000 camera was equipped with a K1 CentriMax long distance microscope lens and was mounted on a Thuralabs breadboard at a safe distance from the high temperature device. The lens included a main body with iris, a Nikon F-mount adapter, a NTX-2x attachment, and a MX-4 objective lens attachment. The complete set-up was successfully operated with the heated chip at temperatures up to $500^{\circ} \mathrm{C}$.

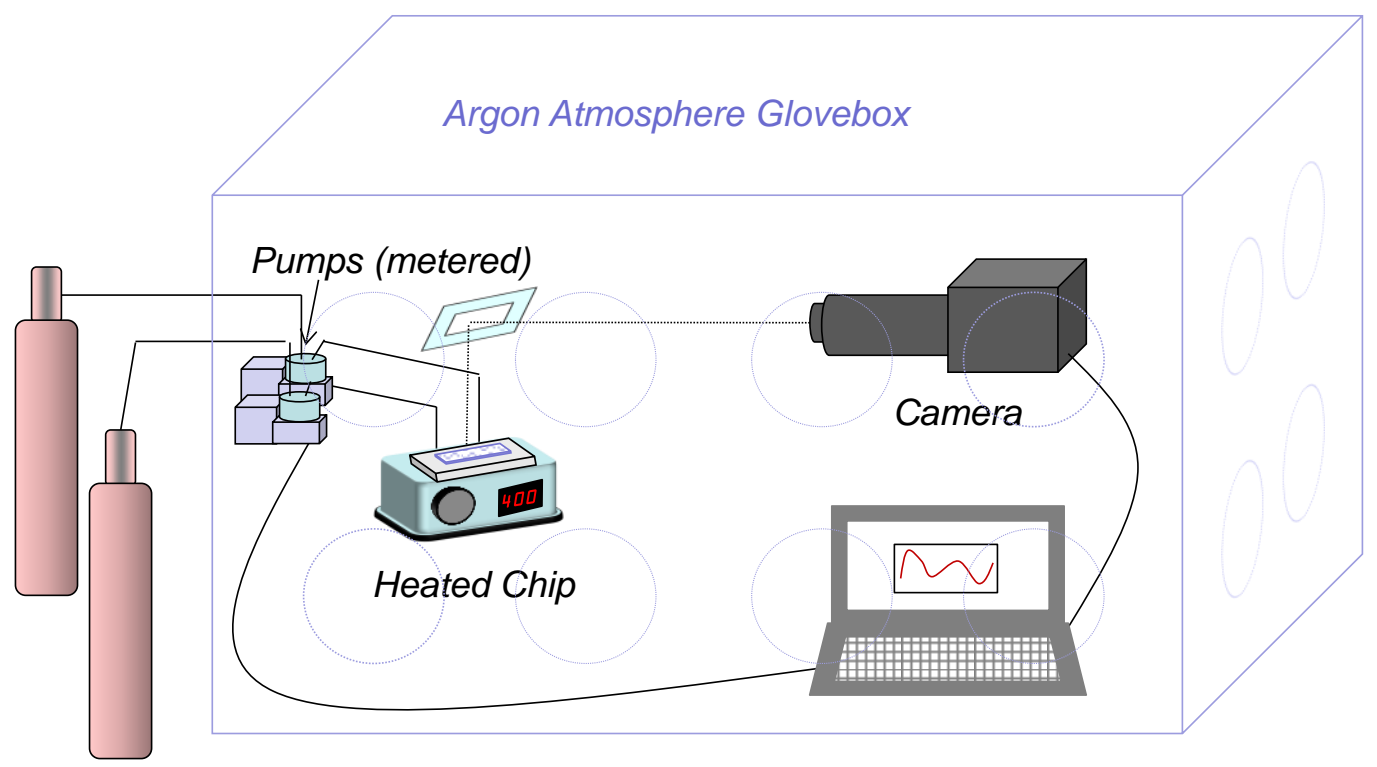

$\mathrm{N}_{2}$ Gas Feed

Figure 5 - Microchip test set-up within glovebox. 
September 26, 2013



Figure 6 - Experimental setup prior to insertion into the inert atmosphere glovebox.

The project was focused on proving feasibility of the concept by testing candidate fluids using the initial chip design. Key characteristic properties for molten salts and water are given in Table 1 . Because the melting point of the chloride eutectic salt is near the manufacturers' stated maximum working temperature for many thermal transfer fluids, a lower-melting halide eutectic salt was selected for initial proof of concept testing. The eutectic chosen $(66 \% \mathrm{LiI}-34 \% \mathrm{CsI})$ has a published melting point of $217^{\circ} \mathrm{C}$, well below the stability limits reported for the heat transfer fluids. Literature values for LiI-CsI fluid properties are not available, however the properties of the $\mathrm{LiCl}-\mathrm{KCl}$ eutectic salt and a nitrate eutectic shown in the Table serve as salt reference points. Water is also included in table 1 for comparison as most of the available literature on slug flows in microchannels is for water-organic systems. It can be seen that the properties of the water are generally intermediate between those of the salts and those of the thermal transfer fluids listed in Table 2.

Table 1: Properties of Molten Salts and Water

\begin{tabular}{|l|c|c|c|c|c|}
\hline \multicolumn{1}{|c|}{ Fluid } & $\begin{array}{c}\text { Density* } \\
\text { (g/cc) }\end{array}$ & $\begin{array}{c}\text { Viscosity* } \\
\text { (cP) }\end{array}$ & $\begin{array}{c}\text { Surface } \\
\text { Tension* } \\
\text { (dyne/cm) }\end{array}$ & $\begin{array}{c}\text { Thermal } \\
\text { Conductivity } \\
(\mathrm{W} / \mathrm{m} / \mathrm{K})\end{array}$ & $\begin{array}{c}\text { Maximum } \\
\text { Temp. } \\
\left({ }^{\circ} \mathrm{C}\right)\end{array}$ \\
\hline${\mathrm{LiCl}-\mathrm{KCl}^{4}}^{4}$ & 1.62 & 1.15 & 102 & 0.42 & $\mathrm{NA}$ \\
$\mathrm{NaNO}_{3}-\mathrm{KNO}_{3}{ }^{5}$ & 1.75 & 6.0 & 110 & 0.40 & $\mathrm{NA}$ \\
Water $^{6}$ & 1.00 & 1.02 & 72.8 & 0.60 & $\mathrm{NA}$ \\
\hline
\end{tabular}

*Value reported for fluid at maximum operating temperature; all other values at $25^{\circ} \mathrm{C}$. 


\section{Testing of a Microfluidic Sampling System for High Temperature Electrochemical MC\&A}

September 26, 2013

Commercially available thermal transfer fluids (TTF) were examined to serve as the carrier phase. Several of these fluids and their characteristic properties are given in Table 2. Duratherm S, HF, and 630 are available from Duratherm Extended Life Fluids, while Therminol 72, 75, and VP-1 are produced by the Eastman Chemical Co. Duratherm S was used in the initial testing. The Duratherm fluid values listed are for the maximum operating temperatures, while the Therminol fluids are properties are for $25^{\circ} \mathrm{C}$ operation. Kinematic viscosity values are listed only for fluids at $100^{\circ} \mathrm{C}$.

Table 2: Properties of Thermal Transfer Fluids ${ }^{7}$

\begin{tabular}{|l|c|c|c|c|c|}
\hline \multicolumn{1}{|c|}{ Fluid } & $\begin{array}{c}\text { Density } \\
(\mathrm{g} / \mathrm{cc})\end{array}$ & $\begin{array}{c}\text { Viscosity } \\
(\mathrm{cP})\end{array}$ & $\begin{array}{c}\text { Surface } \\
\text { Tension } \\
(\mathrm{dyne} / \mathrm{cm})\end{array}$ & $\begin{array}{c}\text { Thermal } \\
\text { Conductivity } \\
(\mathrm{W} / \mathrm{mC}) / \mathrm{K})\end{array}$ & $\begin{array}{c}\text { Maximum } \\
\text { Temp. } \\
\left({ }^{\circ} \mathrm{C}\right)\end{array}$ \\
\hline Duratherm $\mathrm{S}^{8}$ & $0.83^{\mathrm{a}}$ & $2.7^{\mathrm{a}}$ & $0.5^{\mathrm{d}}$ & 0.072 & 345 \\
Duratherm hf & $0.67^{\mathrm{a}}$ & $0.54^{\mathrm{a}}$ & $20^{\mathrm{d}}$ & 0.127 & 335 \\
Duratherm 630 & $0.66^{\mathrm{a}}$ & $0.46^{\mathrm{a}}$ & $50^{\mathrm{d}}$ & 0.128 & 320 \\
Therminol VP-1 & $1.06^{\mathrm{b}}$ & $1.05^{\mathrm{c}}$ & $36.6 \mathrm{a}$ & $\mathrm{NA}$ & 400 \\
Therminol 72 & $1.08^{\mathrm{b}}$ & $6.2^{\mathrm{c}}$ & $\mathrm{NA}$ & $\mathrm{NA}$ & 385 \\
Therminol 75 & $1.04^{\mathrm{b}}$ & $3.9^{\mathrm{c}}$ & $50 \mathrm{~N}$ & $\mathrm{NA}$ & 380 \\
\hline
\end{tabular}

${ }^{a}$ Value reported for fluid at maximum operating temperature;

b Value reported at $25^{\circ} \mathrm{C}$.

${ }^{\mathrm{C}}$ Value reported for fluid at $100^{\circ} \mathrm{C}$

${ }^{d}$ Estimated from values for fluids of similar types ${ }^{9}$

A tube furnace and an MX-1 objective for our long distance microscope were received in FY13 to use for the supplemental measurement of the interfacial properties of the threephase molten salt-thermal transfer fluids-silica system. ${ }^{10}$ Figure 7 illustrates the test set-up for the contact angle measurements to determine the relative surface forces. Because of the high temperature, it is not clear which approach will work best without further testing. The more direct approach is to measure the contact angles formed directly by a modified Sessile drop test, as shown in Figure 7a). A chunk of frozen salt is placed on a plate of VIOSIL-SX synthetic quartz (the same material as the fluidic chip) within a clear quartz cuvette; thermal transfer fluid is carefully added to completely submerge the salt. The cuvette is placed in the center of an alumina tube which is in turn placed in the tube furnace. The entire assembly is heated above the melting point of the salt to form a stable salt droplet on the plate while immersed in the other fluid. The cuvette can be imaged using our long distance microscope and MX-1 objective with the camera aligned with the axis of the alumina tube so that contact angles can be determined from photographic images. The relative surface tensions can be determined from equation 1, which relates the surface forces between the salt-thermal fluid $\left(\gamma_{\mathrm{st}}\right)$ quartz-thermal fluid $\left(\gamma_{\mathrm{qt}}\right)$ and salt-quartz $\left(\gamma_{\mathrm{qs}}\right)$ and the angle between the salt and quartz $(\theta)$. Alternatively the salt can be added via a micro-diameter tube to form a droplet suspended within the fluid. 


\section{Testing of a Microfluidic Sampling System for High Temperature Electrochemical MC\&A}

September 26, 2013

$$
\gamma_{s t} \cos \theta=\gamma_{q t}-\gamma_{q s}
$$

Similarly, the interfacial tensions can be derived from the contact angles formed within capillaries as shown in Figure 7b. Combinations of eutectic $\mathrm{KCl}-\mathrm{LiCl}$, CsI-LiI or $\mathrm{NaNO}_{3}-\mathrm{KNO}_{3}$ and candidate thermal transfer fluids will be placed in crucibles inside a furnace well inside a glovebox. Miniature, small bore steel tubing or fused silica tubing will be inserted into each crucible. The tubing will be connected at a t-junction, inside the furnace well. The furnace well atmosphere will be the dry and inert (Argon) glovebox atmosphere. The furnace temperature will be controlled using existing thermal control systems associated with the glovebox. The furnace will be heated to $400^{\circ} \mathrm{C}$. A moderate vacuum will be applied to the room temperature side of the tubing, drawing the mixture of eutectic $\mathrm{KCl}-\mathrm{LiCl}$ and thermal transfer fluids out of the furnace. Once the liquids reach the freezing point on the room temperature side, flow will stop. The glass capillary with the frozen phases will be removed from the glovebox and examined under a microscope.

Additional useful design information can be collected by studying the dynamic contact angles within a microfluidic chip under static and flow conditions. Combinations of eutectic $\mathrm{KCl}-\mathrm{LiCl}, \mathrm{CsI}-\mathrm{LiI}$ or $\mathrm{NaNO}_{3}-\mathrm{KNO}_{3}$ and candidate thermal transfer fluids will be run through our droplet generating device (as described in section 4) and contact angles will be measured via imaging and automated image analysis. The flow rate will be varied (including stopping the flow) to elucidate the effects of fluid velocity on the dynamic contact angles within our specific geometry.

a)

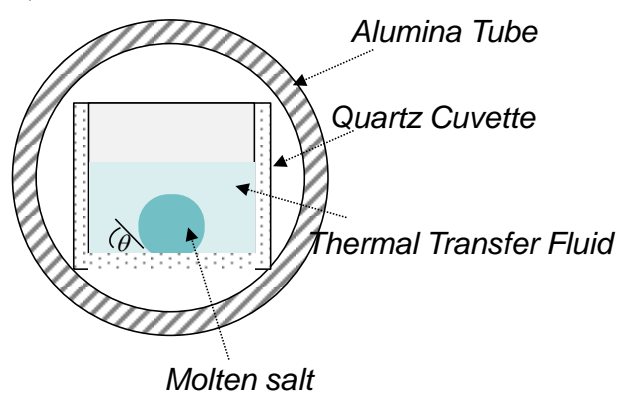

b) Thermal

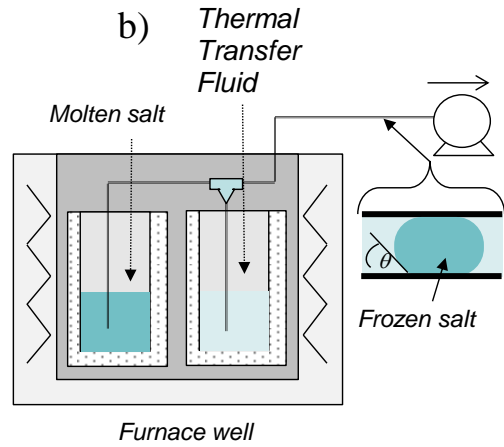

Furnace well

Figure 7 - Conceptual set-ups for droplet tests (a) static and (b) flowing.

The temporary shut-down of laboratory work in building 205 did not allow experimental work after March 29, however, initial testing indicated that the microflow and imaging systems work as currently configured. Multiphase flow, including flowing droplets, was observed, though the contrast between phases was poor and the in-channel freezing needs refinement if uniform frozen droplets are to be produced. The major components required for the contact angle tests were obtained but the equipment has not yet been installed. Overall, the initial tests demonstrated the feasibility of the concept and utility of the current design. 


\section{Testing of a Microfluidic Sampling System for High Temperature Electrochemical MC\&A \\ September 26, 2013}

\section{Testing}

LiI-CsI salt in powder form and Duratherm S TTF were added to the feed reservoirs located on microchip prior to placement in the holder assembly. The chip was then carefully located within the holder and sealed with a graphite gasket. The hot plate was heated to approximately $400^{\circ} \mathrm{C}$ to ensure a chip temperature above that of the melting point of the salt and below the operating maximum of the TTF. Once the chip was up to temperature and the salts melted, the nitrogen gas manifold pressure was set to that specified by the pump manufacturer. Once the system was at pressure the inlet pressure was adjusted until stable flow of both fluids was obtained. Figure 8 shows a schematic of the microchip/inlet fluid flow configuration. The goal was to attain relative flows such that uniform salt droplets (slugs) are formed at the inlet junction that then proceed to flow smoothly in the channel carried along by the TTF. The effect of cooling the chip on the salt forms within the channels was also noted, as most detector systems operate at ambient temperatures.

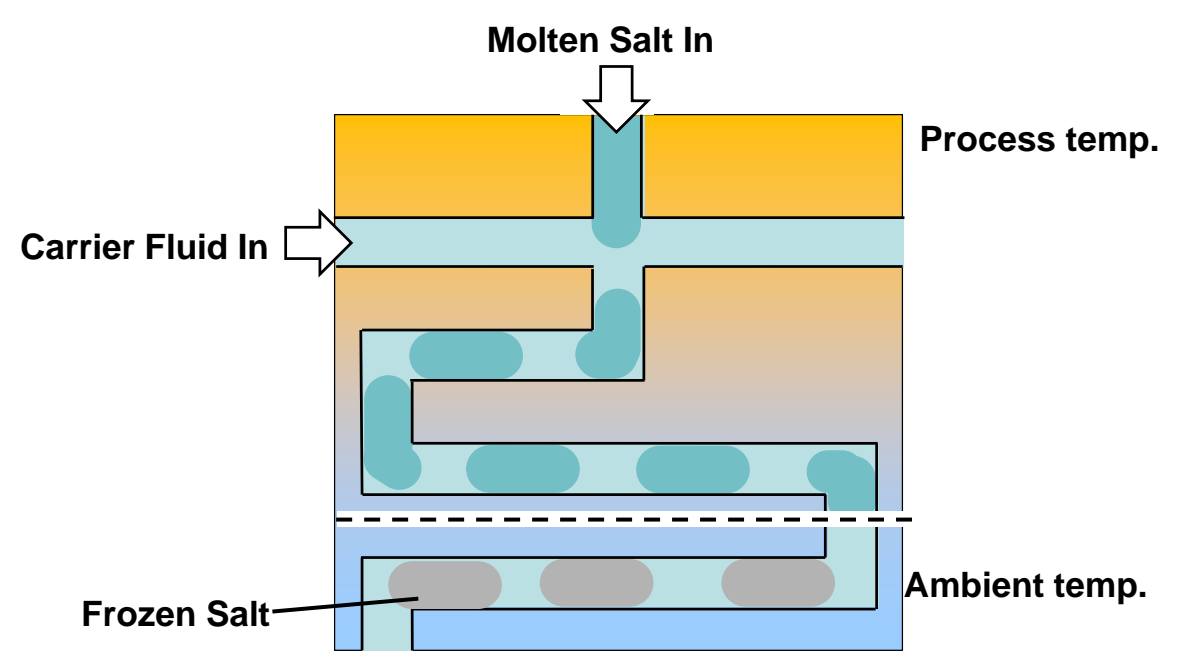

Figure 8 - Schematic of desired fluid flow within the microchip.

The initial tests were run to test the effectiveness of the chip holder and the first iteration of the chip design. The system was heated until a thermocouple inserted into the holder registered $400^{\circ} \mathrm{C}$. At this setting the salt remained molten and there was no notable degradation of the TTF based on observation as the TTF did not appear to darken, evolve gas, or become gummy for the short duration tests; no extended experiments were run so minor degradation cannot be completely discounted. Targeted tests of the TTF stability would be done without the chip in a manner similar to the contact angle tests described above rather than in microchips where such effects would be difficult to measure.

Tests were run with a $66 \mathrm{~mol} \%$ lithium iodide $-34 \mathrm{~mol} \%$ cesium iodide eutectic salt mixture as the intended droplet phase and Duratherm $S$ thermal transfer fluid as the intended carrier phase. The formation of slug flow has been shown to depend on the Weber Number 


\section{Testing of a Microfluidic Sampling System for High Temperature Electrochemical MC\&A \\ September 26, 2013}

(We), given by equation (2) below, which relates the magnitude of the inertial forces to the interfacial forces of the fluids.

$$
W e=\rho v^{2} d / \sigma
$$

Here $\rho$ is the fluid density, $v$ is the linear velocity, $d$ is the channel diameter and $\sigma$ is the interfacial tension between the two fluids. Figure 8 shows the dependence of We on fluid flow rate for several different fluids for two different microchannel diameters. Jovanovic and co-workers ${ }^{2}$ observed that slug flow was predominant when the Weber number was at a value of less than 0.1 for both the organic and aqueous phases in immiscible two-phase flow. The occurrence of slug flow was dependent on the length of the channels, with the onset of parallel or bubbly flow generally observed for smaller Weber numbers in shorter channels. At large We values $(>1)$ for either phase, the flow structure varied considerably (parallel, annular, bubbly, etc.) but consistently for the systems examined.

Because the final system will use LiCl-KCl, and relatively little data was available on the LiICsI eutectic used in the initial salts, the initial chip design was based on the characteristics of LiCl-KCl. As can be seen from the data in Table 1, both the chloride and nitrate salts would be expected to yield similar We values and thus similar flow behaviors within the channels of the microchip since the surface tensions and densities are comparable. It was assumed for the initial flows that the iodides would have properties similar to those of the chlorides and thus demonstrate similar behavior.

Based on the preliminary trials it is clear that the concept of establishing stable two-phase flows in microchannels at elevated temperatures is feasible. Stable flow was established for several different flow conditions. However, the desired flow characterized by consistent droplets of molten salt within a TTF carrier phase could not be verified. A number of different flow patterns, consistent with the flows identified by Janovic and coworkers ${ }^{2}$ were observed including flow consistent with two phase slug flow. Several of the observed characteristics are discussed in the following sections.

One of the readily observed difficulties in characterizing the flows was the poor contrast between the molten salt and TTF phases. Figure 9 is illustrative of this problem. In the figure, the flowing solution can clearly be discerned from the background metal. The synthetic quartz microchip is transparent in the visible and UV range. The unpolished surface of the stainless steel chip holder is visible in the background as series of thin parallel abrasion lines. In some locations large scratches and splotches are also evident on the holder surface in the background. In contrast, the liquids appear relatively dull and somewhat opaque. Although difficult to see, there appear to be two liquid phases as evidenced by the curved edges of the droplet-like forms in several locations in the microchannel. One such form can be seen in the Figure 10 at the far right. Although these "droplets" can be roughly identified, it cannot be determined whether they are clearly salt, TTF or entrained nitrogen, although the latter would be expected to yield relatively clear bubbles that are generally easier to distinguish from liquid phases. 
Testing of a Microfluidic Sampling System for High Temperature Electrochemical MC\&A

September 26, 2013
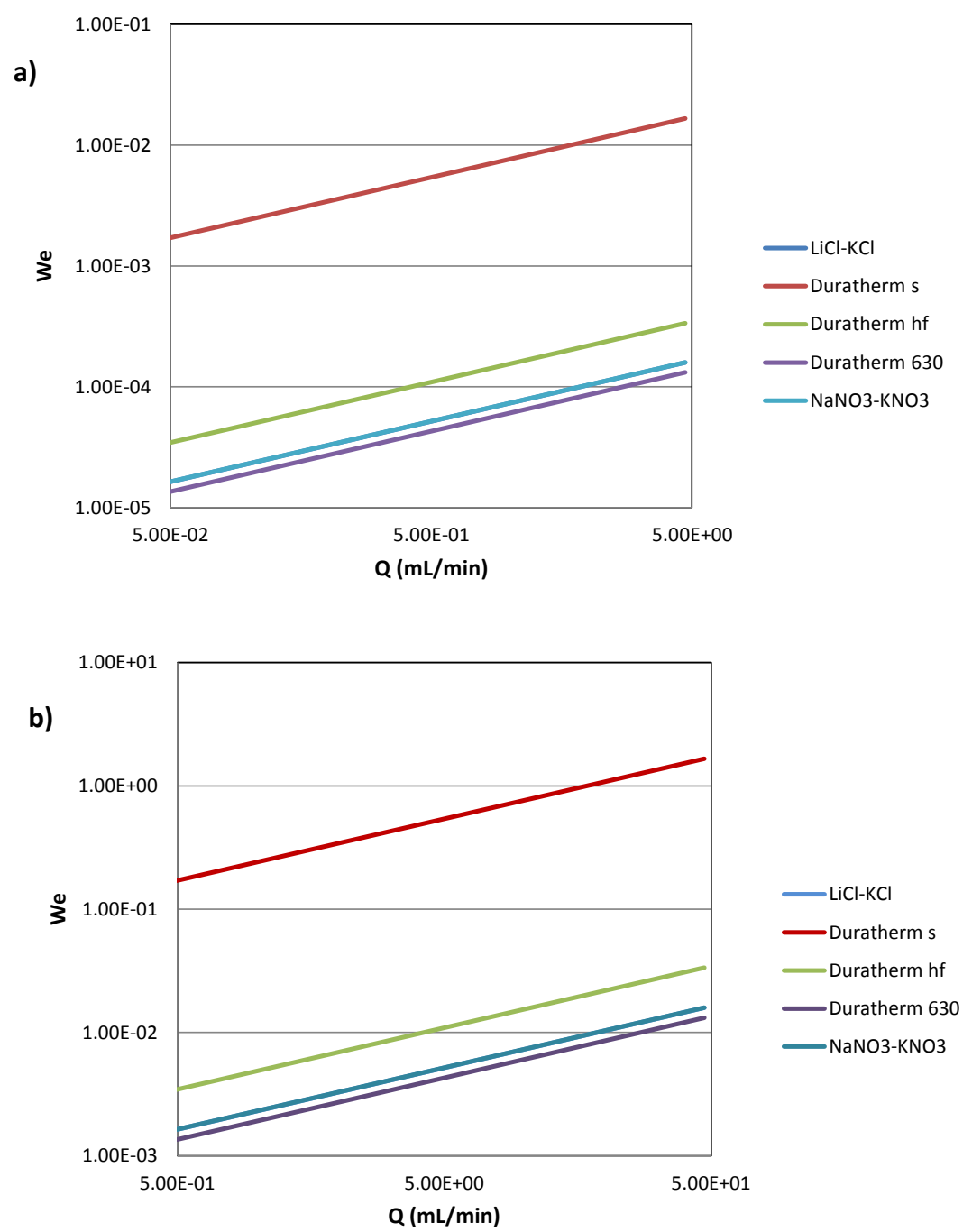

Figure 9 - Dependency of Weber number as function of flow rate for different fluids at two linear velocities (a) low value and (b) higher value. The molten salts curves essentially overlap.

One of the development efforts going forward is to improve imaging by increasing the contrast between the two liquid phases. As a number of candidate TTRs remain to be tested, all differing in composition and properties, it is possible that stronger contrast is available, for example a TTR with a higher index of refraction or strong color. A perhaps more promising option is to add colored salts to the eutectic mix. Lanthanides would appear to be the optimal choice as these are a significant source of salt-soluble fission products and therefore would be present in the process salt for which the sampling system would be deployed. It may be possible to take advantage of not only the color, but also the strong fluorescence of some lanthanides such as Eu and Tb. Flourscent imaging can be carried out with our current imaging system with a few modifications. Imaging the salt droplets fluorescently would simplify image processing, allowing for automated high throughput analysis of droplet sizes. An additional benefit of imaging the system fluorescently is that 


\section{Testing of a Microfluidic Sampling System for High Temperature Electrochemical MC\&A \\ September 26, 2013}

unwanted lanthanide leaching into the carrier fluid could be detected. Furthermore, because the fluorescent intensity of lanthanides is a function of concentration and temperature, a quantitative analysis of droplet images could be used to monitor the spatial and temporal variation of these parameters in future studies.

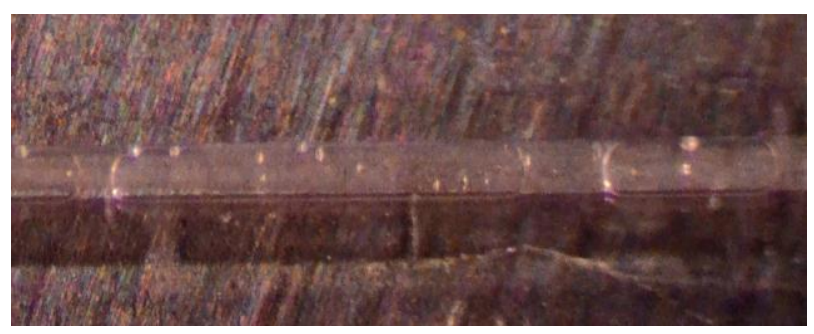

Figure 10 - Photo of microchannel section showing the apparent two-phase fluid in flow.

Another effect observed during imaging, and especially evident during filming, was a vibration or notable shimmer. There are two factors that contributed to this effect. The glovebox itself has a slight vibration associated with the rotary vane pumps used to maintain a vacuum in the glovebox's two transfer locks, and the blowers in the Vacuum Atmospheres Dri-Train ${ }^{\circledR}$ that circulate the glovebox atmosphere. The second source of shimmer, as opposed to vibration, arises from the steep temperature gradients in air between the chip surface and the mirror a few inches above the chip. This shimmer in particular affects the sharpness of the images collected as movies or at extreme close-up as in Figure 10, and is most problematic with low contrast fluids. High-speed photos were generally sharper. Because they are intrinsic to the operation of the glovebox and the chipholder systems, it is unlikely that the effects can be reduced dramatically. However, it should also be noted that neither of these effects impedes imaging drastically as the two phases can be discerned in cases with appropriate lighting as seen in the various photos in this report. Rather, they put a premium on sharpening the contrast between the phases. The slight blurring of the droplet near the inlet cross junction in Figure 11 serves as an example of the impact on filming associated with the external vibration.

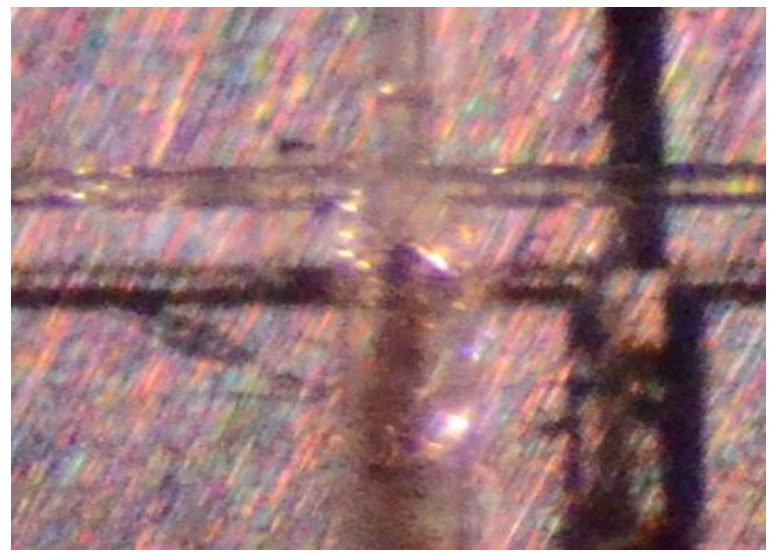

Figure 11 - Photo of inlet cross-junction showing inflows of salt (top) and carrier fluid 
Testing of a Microfluidic Sampling System for High Temperature Electrochemical MC\&A

September 26, 2013

(sides) and resulting two-phase emulsion (bottom).

For nearly all of the fluid flow rates that were tested, stable, consistent salt droplets were not formed. The most commonly observed phenomena were premature breakup of droplets leading to formation of parallel, wavy, or annular flows. Examples of each of these cases can be seen in Figures12a) and 12b) for two different TTR flow rates. A good illustration of the formation of these flows is seen in Figure 13, which shows fluid from the cross-junction where the two fluids meet to the first bend of the microchannel. In Figure 13 a), a droplet of salt appears to form just past the junction. The droplet continues to grow until the point in Figure 13b) where the leading edge breaks before the trailing edge disengages from the cross-junction. In Figure 12c), a new salt droplet begins to form once the prior drop disengages. At the lower left, the onset of a wavy flow pattern is evident as the fluid continues to flow (left). To form stable droplet the tailing edge must disengage before the leading edge breaks. Several options may be pursued including increasing the TTR flow rate, changing the relative surface tensions, or changing the channel dimensions and/or length, or inlet geometry. Only changes in flow rates have been tested to date.
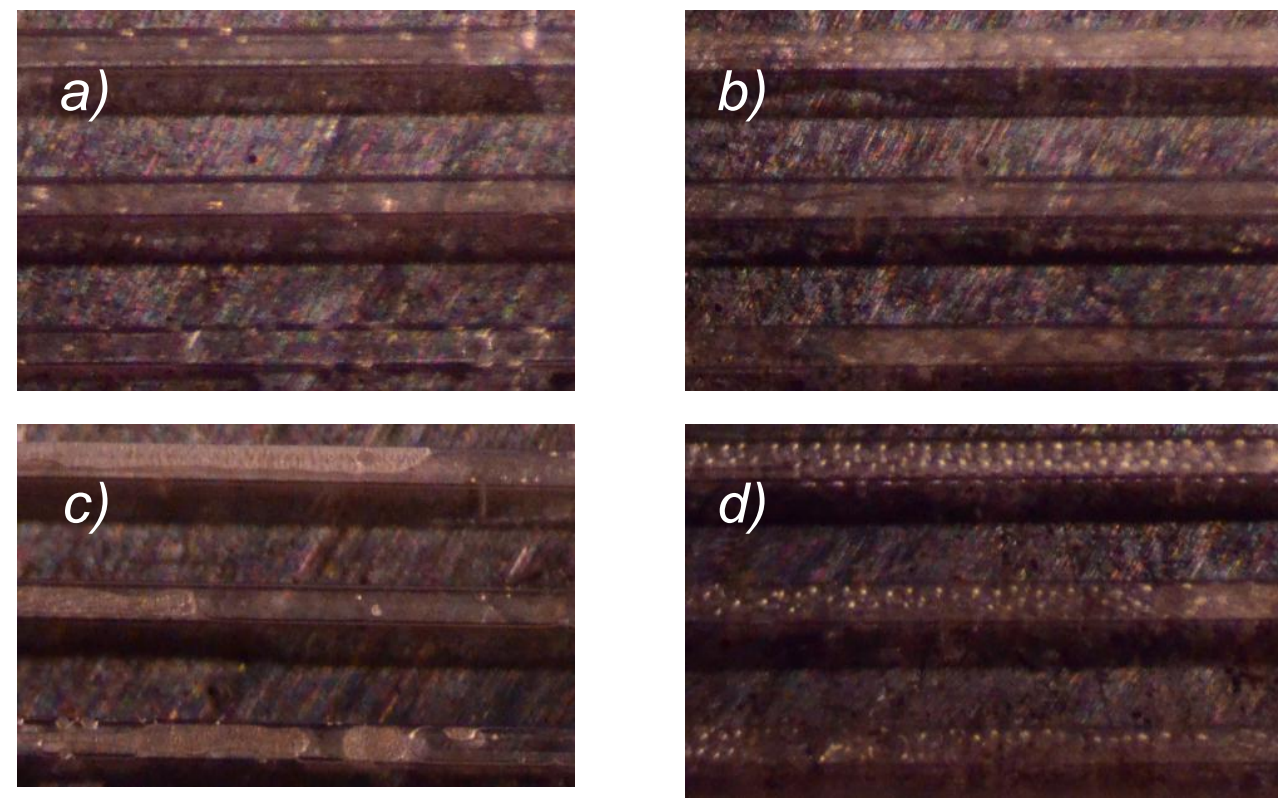

Figure 12 - Set of photos showing different mixed flows of molten salt and heat transfer fluids within a microchannel (a) annular, (b) wavy parallel flow, (c) foamy solid salt (not flowing), and (d) bubbly flow. 


\section{Testing of a Microfluidic Sampling System for High Temperature Electrochemical MC\&A}

September 26, 2013
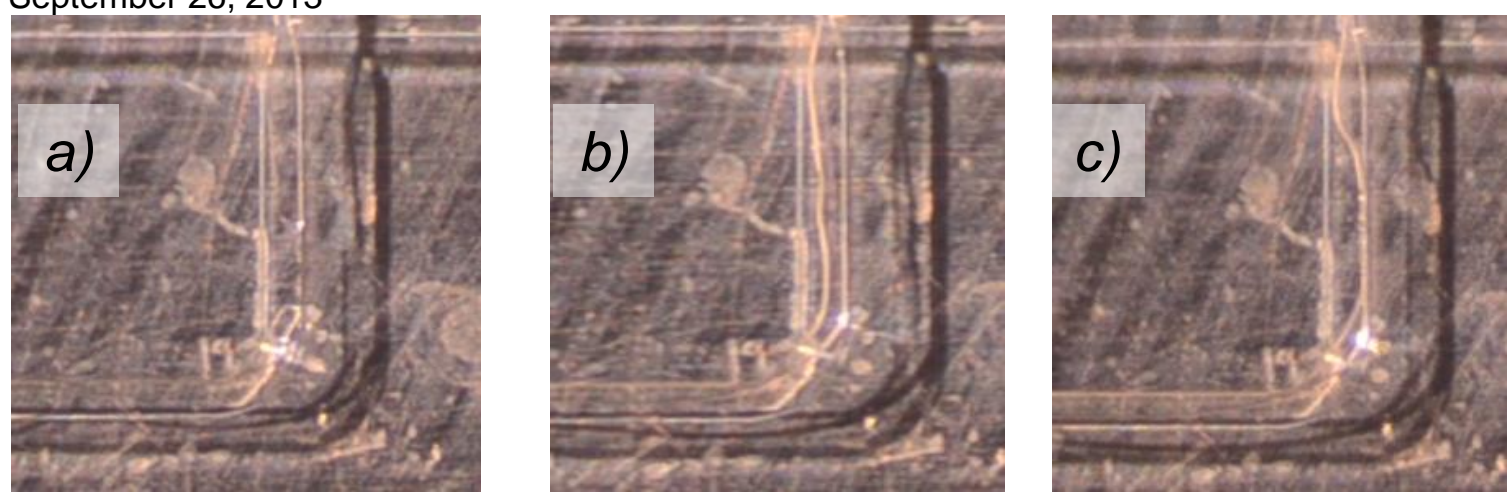

Figure 13 - Set of photos showing flow progression at inlet junction of salt (from top center) combining with heat transfer fluid (from top left and right) to form two phase wavy parallel flow (downward and bottom left).

As most detectors and analysis systems operate at near room-temperature, one aspect to be determined is if the droplets can be frozen within the channels. As the salt should contract slightly, if sized correctly the solidified droplets can be moved along to the channels by the carrier fluid to the cooled zone where they may be analyzed individually spectroscopically, by LIBS, alpha or gamma spectroscopy, or another technique. Room temperature microfluidic devices for processing and transporting solids suspended in carrier fluid are well characterized,11 and therefore similar configurations would be adapted to this application with TTF fluids carrying entrained solid salt slugs.

Included inthese tests was a first study of salt freezing within the channels. After flow tests, of necessity the microchips were allowed to cool while filled with both fluids but with no flow. The resulting salt structure tended to vary significantly as can be seen in Figure 13c) and Figure 14. Figure 13c) shows an immobile extended salt structure somewhat consistent with the extended wavy parallel flows of Figure 13b). In some cases, salt froze into extended "droplets" as can be seen in Figure 14. Although it is these types of droplet formations that are targeted, those that were observed were not dimensionally consistent, varying from small individual capsule-shaped pieces to more extended capsule-like structures. As the fluid flows were restarted, droplets or bubbles appeared to form, suggesting either nitrogen gas was being entrained or uneven melting of the salt phase. Figure 12d) shows the region of Figure 12c) after reheating and restart of fluid flows. The salt darkens (melts) and appears to move. The frozen salt that resulted from shutting down the experiment was removed effectively in this manner on restart. Salt could also be removed from the channels by immersing the chip in a water bath. Once the operating conditions that result in regular slug flow are determined, freezing of the salt phase within the microchip will be studied more closely so that a working procedure for shut-down, cooling, and restart of flow can be developed. 


\section{Testing of a Microfluidic Sampling System for High Temperature Electrochemical MC\&A}

September 26, 2013

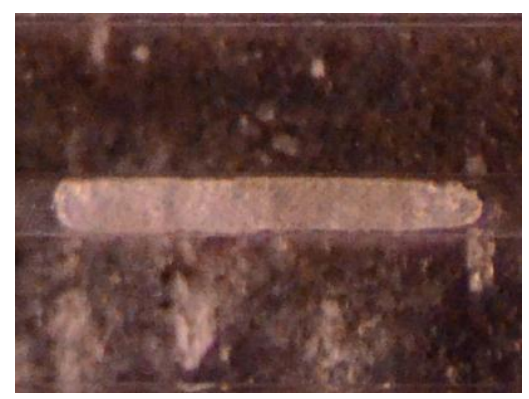

Figure 14 - Photo of frozen salt "droplet" within microchannel system.

By adjusting the inlet nitrogen gas pressure, and thus changing the relative flow rates of the two phases, an apparent two-phase slug flow was achieved. Figure 14 shows what appears to be clear two-phase slug-type flow within the chip system. The droplet dimensions and inter-drop distances were generally, but not universally, consistent, as can be seen in the figure for two of the three parallel channels. Uncertainty arises from the high contrast visible between the two phases (compare with Figures 9 and 11), which is not evident in other tests, and the discrepancy between uniform slug flow in the lower two channels and an absence of slugs in the upper channel. Intermittent entrained gas slugs were evident in the salt phase channels above the cross-junction (i.e. prior to mixing of the two fluids), but it is not clear why regular elongated bubbles would have formed or why only one liquid phase can be discerned. Though movement of the entrained gas slugs above the cross-junction confirmed that the salt was advancing, no such bubbles were evident in the TTR phase above the junction. One possibility is that the TTR phase degraded sufficiently to halt its flow while allowing nitrogen to pass through. In any event, this result does demonstrate that slug flow is attainable for molten salts in microchannels under the correct flow conditions.

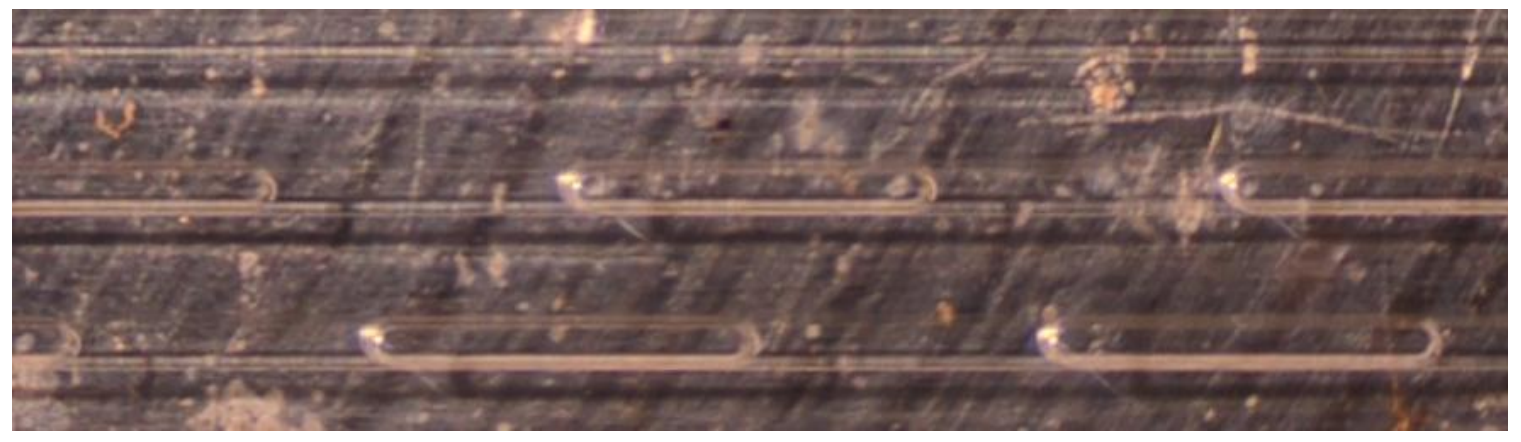

Figure 15 -Photo showing two-phase slug flow in three microchannels. Consistent flow is evident in bottom two channels. Clear interfacial delineations suggest gas-liquid rather than liquid-liquid mixed flows. 
Testing of a Microfluidic Sampling System for High Temperature Electrochemical MC\&A

September 26, 2013

\section{Conclusion}

This report described the initial set-up and testing as well as future plans for microfluidic sampling systems for monitoring electrochemical processes to support material balance and control systems in a used fuel treatment facility. An experimental apparatus was also assembled to measure contact angles and relative surface tensions for a variety of liquids relevant to the on-line monitoring platform.

In FY13, the first test microchip and holder operated with characteristic fluids at nearprocess temperatures $\left(\sim 400^{\circ} \mathrm{C}\right)$. The assembly is being used to elucidate the behavior of the molten salts and solids in microchannels. Tests were run with a LiI-CsI low-melting eutectic salt and a thermal transfer fluid as the carrier. Several additional candidate thermal transfer fluids will be tested to determine their suitability as material transfer media. The initial tests were intended to optimize operating conditions for each liquid-liquid system in order to characterize their properties and behavior under process conditions. The microchip imaging set-up was found to be capable of imaging the high temperature chip from a safe distance. However, due to poor contrast between the fluids, further effort to examine contrasting agents that can be added to a liquid component and a refining the imaging techniques is required. Additional testing of the chip and holder set-up at near-process temperatures is required to obtain the data necessary to better tailor future iterations of microchips to the LiI-CsI and LiCl-KCl systems. Although a number of flow types were observed, including the desired slug flow, the establishment of stable flow and consistent droplets must be verified, while the contrast between phases and in-channel freezing of salt should be improved. As testing proceeds, the chip design will be refined and procedures developed for cooling and heating microchips.

Once the concept is more fully developed for a single temperature zone, the approach can be used in designs with multiple temperature zones, which will likely be required in the deployed design. Any necessary refinements will be made once a gauge of the performance is attained. In the longer term, it will be necessary to develop an interface with on-line/atline analysis equipment to assess temperature and timing requirements, as well as purification requirements. All multi-zone modifications will require detailed design specification for fabrication and eventual testing. Integration of purification with thermal transfer requirements and stability in high-radiation, high-temperature environments must all be demonstrated.

\section{References}

${ }^{1}$ Zhao, C-X and A.P.J. Middelberg, Chem. Eng. Sci., 66, 1394 (2011)

${ }^{2}$ Nichols, K. P., et al., J. Am. Chem. Soc., 133 (39), pp 15721-15729, (2011)

3 Jovanović, J., et al. ,Ind. Eng. Chem. Res., 51 (2), pp 1015-1026, (2012)

${ }^{4} \mathrm{LiCl}-\mathrm{KCl}$ reference 
${ }^{5}$ NaNO3 reference

${ }^{6}$ Water properties reference

${ }^{7}$ Therminol Website www.therminol.com

${ }^{8}$ Duratherm website www.heat-transfer-fluid.com

${ }^{9}$ Katz, D.L and W. Saltman, Ind. And Eng. Chem, 31(1), 92 (1939); L.A. Girafalco and R.J. Good, J. Phys. Chem, 61, 94 (1957); D.E. Hirt, et al., Colloids and Surf. Sci., 44, 101 (1990).

${ }^{10}$ Eustathopoulos, N., et al., J. of Mat. Sci., 40, 2271 - 2280, (2005)

${ }^{11}$ DiCarlo, D., et al., Phys. Rev. Let., 102, 094503 (2009) 